\title{
FORMAL MODELLING AND SIMULATION OF A MULTI-AGENT NANO-ROBOTIC DRUG DELIVERY SYSTEM
}

\author{
MARINA NTIKA, PETROS KEFALAS, AND IOANNA STAMATOPOULOU $\ddagger$
}

\begin{abstract}
Targeted drug delivery with the use of nanorobots, a yet mostly theoretical but very promising future concept, is anticipated to become a significant ally in cancer treatment. The way that nanorobot systems are currently envisaged by researchers is such that they exhibit autonomous and collaborative behaviour that can be uniquely captured by multi-agent systems. In this paper, we investigate this hypothesis by describing the process of formally modelling a simple agent-based system for a simulation of targeted drug delivery. We propose a system comprising different types of nanorobots, and evaluate the effects of various parameters on the final outcome. The data that were retrieved from the corresponding simulation runs, are in support of our hypothesis, demonstrating that nanorobotic drug delivery systems can be effectively simulated by utilising intelligent agent technology.
\end{abstract}

Key words: multi-agent system, simulation, drug delivery, nanorobots, formal modelling

AMS subject classifications. $68 \mathrm{~T} 42,68 \mathrm{U} 20$

1. Introduction. Multi-Agent Systems have established their applicability in studying complex systems that involve a number of heterogeneous resources working collaboratively towards solving a common problem, despite the fact that each individual might have partial information about the problem and limited capabilities. As stated by Brooks in [4, 3], Multi-Agent Systems (MAS) may potentially exhibit "emergent intelligence" commonly found in biologically inspired systems.

In parallel, Nanotechnology, currently one of the most important and fast growing areas in modern science, focuses on manipulating matter with dimensions similar to the ones of biological molecules. Many scientific fields, such as Physics, Material Sciences, Chemistry, Chemical Engineering, Microbiology and Genetics, are currently enjoying the fruits of nanotechnological research [10], which has already led to various commercially available applications, but what appears to be amongst the most promising endeavours is the development of nanotechnological constructs targeted for medical use. One quite attractive but also challenging such application is the targeted drug delivery, which is anticipated to be a revolutionary strategy for overcoming serious barriers in health impairments, and nowadays, with the rapidly increasing nanotechnology advances, is regarded as one of the most promising research topics worldwide.

Combining the aforementioned research areas, our contribution focuses on the formal modelling and development of a MAS simulation of nanorobots that deliver an appropriate pharmaceutical substance to unhealthy human body tissue. Inspired by the fact that in silico experimentation has established its importance to the research community over the past decade, with mathematical models, algorithms and computer simulations having proven to be valuable allies in studying various and diverse problems in the fields of Biology and Medical Sciences [31, 24], we opted to simulate a simple targeted drug delivery system.

The paper is organized as follows: Section 2, briefly discusses the current status of nanotechnology in medicine and the way computer simulations are employed in medical nanorobotics, with special focus on MultiAgent System simulations. In Sections 3 and 5 our proposed system is presented and the formal modelling, simulation design and implementation processes are described. Section 6 includes the experimental analysis and the results of the simulation runs. In Section 7 the contemporary approaches in manufacturing robots in the microscale are presented, and finally, we conclude with a short discussion for further work in Section 8 .

\footnotetext{
* South-East European Research Centre (SEERC), Research Centre of the International Faculty of Sheffield, CITY College, 24 Proxenou Koromila Str. 546 22, Thessaloniki, Greece(mantika@seerc.org).

†The University of Sheffield International Faculty, CITY College, 13 Tsimiski Street, 54624 Thessaloniki, Greece(kefalas@city.academic.gr)

$\ddagger$ The University of Sheffield International Faculty, CITY College, 3 Leontos Sofou, 54626 Thessaloniki, Greece(istamatopoulou@city.academic.gr)
} 


\section{Background and Similar Work.}

2.1. Nanotechnology in a Nutshell. The prefix "nano-" means a billionth, hence, when it is added in front of the word "meter" it denotes a factor of $10^{-9}$, resulting in dimensions comparable to atomic diameters. Consequently, Nanoscience studies the properties of objects at the nanoscale, and Nanotechnology focuses on manipulating matter in structures with at least one dimension sized from 1 to 100 nanometers. Both fields have a multidisciplinary core, involving researchers of diverse backgrounds and with variant skills. Current advances in the domain have been receiving much attention for the past two decades, both from the Academia and the Industry, largely due to the fact that nanostructures exhibit unique properties and characteristics [29, 26, 53], profoundly different than the ones that have been observed at the macroscale.

A plethora of applications in a wide range of fields are currently available, spanning from flexible digital screens to self-cleaning surfaces, from nanoparticle comprised catalysts [48] to carbon nanotubes acting as chemical sensors [51] etc. However, the potential of nanotechnological constructs targeted for medical use is of special interest.

Nanoparticles, being considered as the building blocks for nanotechnology, are of great scientific interest, due to the unique properties they often possess. Those properties are mostly attributed to the far larger surface area of nanostructured materials compared to masses built from larger-scale blocks. From a biomedical viewpoint, their size is what makes them a very attractive option for a variety of applications, among which drug-delivery and biological and chemical sensing being the most predominant ones. When used for drug-delivery systems, the nanoparticles carry a payload that consists of an appropriate pharmaceutical substance or other types of payload, i.e. proteins, genes etc. This payload is encapsulated in a biocompatible and possibly biodegradable coating. Due to their small size, nanoparticles can evade immune system detection and cross the blood-brain barrier gaining access to the brain. Nanoparticles suitable for drug-delivery, such as dendrimers, nanocrystals, polymeric micelles, lipid nanoparticles and liposomes [15] are already being manufactured. Those nanostructures exploit their inherent biological characteristics, and are based on molecular and chemical interactions to achieve the target-specific delivery or their biochemical sensing. In in-vitro experiments [25, 43], the target recognition is performed by administering these nano-vehicles into a cell-culture or some other suitable substrate, whereas in in-vivo $[27,16]$ experiments the nanoparticles are injected into the area of interest, either the bloodstream or at specific animal body sites. The nanoparticles can only recognize their target and bind to it when they randomly come in contact with it. Additionally, the process of releasing their payload lies purely on the rate by which their protective surface biodegrades.

Notwithstanding the fact that research in this domain is still in its infants, scientists already envision a far more effective schema, where the nanodevices will attain additional properties, such as communication and navigation. These nanodevices can be also characterized with the term "nanorobot".

2.2. Simulation in Medical Nanorobotics. Although a plethora of nanotechnological applications are already commercially available [34], what is considered as a fundamentally important sector, human health, is yet to enjoy substantial benefits. This is partially due to the very nature of medical research, which requires lengthy periods of time, usually at least a decade, and adherence to strict protocols, before any new developments are encompassed. Under these circumstances, computer simulations offer a great advantage towards accelerating both the basic and applied research processes.

Computer simulations can offer an advantageous insight into medical nanorobotics. On one hand, appropriately designed simulations provide a method for thoroughly investigating the properties and dynamics of virtually any conceptual system. Data can be collected and possible behavioural patterns may be discovered, even before the physical elements comprising the system are technologically available. On the other hand, such simulations can produce valuable information on the way those physical elements must be built.

Recognising the beneficial implications of biomedical computer simulations, a number of studies have been published during the last decade. Unmat et al. [52] discuss the use of Molecular Dynamics, Molecular Kinematic and Monte-Carlo simulations to assist in predicting the performance of bio-nanocomponents on a molecular level, such as energy and force calculations, calculating all feasible conformations of a biomolecule etc. Sharma et al. [46] discuss the potential of combining Molecular Dynamics simulations with Virtual Reality to portray more accurately complex molecular structure models, that include unique nano-properties such as interatomic forces, electrostatic fields around molecules etc. 
Going further to study the possible coordination and control of a swarm of nanorobots that are inserted in body tissue, Calcavanti et al. have developed the Nanorobot Control Design (NCD) simulator [5]. In [5], the NCD simulator is used to investigate a fractured coronary artery. The environment of the fractured artery was initially simulated using FLUENT, a finite-volume based Computational Fluid Dynamics (CFD) package [14]. The simulation in FLUENT produces a set of output data, such as the temperature heterogeneity that is known to exist over inflamed plaque surfaces. Those data are subsequently fed as input to NCD, along with several other parameters produced by FLUENT, i.e. blood flow velocities in different parts of the arteries, cytokines and adhesion molecule concentrations that are of interest etc. NCD then produces the visual representation of the nanorobots operating environment. The authors demonstrate the applicability of NCD by using it to determine the optimal values of trigger events that would cause a nanorobot to perform its pre-defined task. The nanorobots, as envisioned by the authors, comprise molecular sorting rotors and robotic arms, biocompatible exteriors and chemotactic sensors for distinguishing between different molecules and detecting possible obstacles in their route.

NCD is used to further simulate other nanorobotic systems for medical applications, such as the potential benefits that could rise by using nanorobots in laparoscopic cancer surgery [8]. Nowadays, the use of sophisticated robotics machinery in laparoscopic cancer surgeries is widespread, with the daVinci surgical system [17] being the cutting-edge technology used. DaVinci comprises a surgeon console, a patient-side cart and an imageprocessing or insufflation stack. It is proposed by the authors to add nanorobots as a component, in order to achieve advanced high-precision mapping of cancerous areas that the surgeon must dissect. CMOS manufacturing technology is suggested, with addition of chemical sensors to detect chemical changes. The authors' simulation focuses on the chemical signal detection capabilities of 50 nanorobots inside a vessel, and on the manner that the vessel size and the relative position of the nanorobots to the vessel walls can affect measurements. Additional NCD simulations may be found in $[6,7]$.

Following this brief discussion on the impact that computer simulations may have on refining the characteristics of future nanorobots, it is purposeful to investigate what type of model is appropriate for capturing the dynamics of such a system.

2.3. Nanorobots as a Multi-Agent System. The characteristics that future nanorobots for medical applications should possess are described in [38], to include biocompatibility and power to function, communication and navigation. A variety of nanorobotic design and control required functionalities is discussed in [52], including amongst them swarm behaviour and bio-nanointelligence. The authors attribute the capability of making decisions to the latter term. It can be therefore stated that, overall, nanorobot coordination is of paramount importance, as nanorobots will need to coordinate their actions in a decentralized manner, to behave cooperatively, to be programmable and able to process information, all combined towards achieving a common goal.

Setting aside the issue of biocompatibility, which falls mainly under the scope of biological research, the remaining features, namely power supply, communication, navigation and coordination, have to be considered when selecting the software approach that would be suitable for modelling a system comprising nanorobots. Such a system can be conceptualized as a number of autonomous entities, each having own individual features and properties, which work collaboratively towards achieving a common goal. Following this line of reasoning, a multi-agent system appears to be an appropriate approach that captures this type of collaborative behaviour.

Holland and Melhuish [19] focused on investigating minimal agents' systems, by simulating agents with minimal sensing, mobility and computational capabilities either homing a static beacon or following a moving one. Several algorithms are implemented and compared, with or without agent interaction, in an effort to study the possible collective behaviour of micro- and nano-robots. One of the interesting findings in this work is the capability of secondary swarms in tracing the attraction source. Towards that direction, Melhuish and Welsby in [32], specifically studied the impact of secondary swarming by implementing some of the algorithms from [19] in real blimp robots that were carrying $96 \mathrm{~g}$ of Helium as a payload, and found significantly increased performance in homing a static beacon when incorporating collective behaviour compared to employing purely individualistic strategies.

A multi-agent system simulation is used in [41] that includes an indirect communication model (i.e. agents with no direct communication but able to communicate with a base station), and to investigate its effect on the 
efficiency of locating a target within a complex and dynamically changing environment, comprising biological cells that deform over time. A multi-agent simulation is designed for cancer monitoring using nanorobots [42]. Using NetLogo [54] three simple bio-inspired strategies of nanorobot target finding in complex fluid environments were implemented and results are presented by comparing the average times of locating the target. The results of the ran experiments indicated that all strategies were effective in locating the target within the pre-set time limit, with the "wandering" strategy, i.e. aimlessly moving agent, adjusting direction when it senses the target, producing the best finding times.

3. A Nanorobotic Targeted Drug Delivery System. In [37] we presented a simulation of a number of nanorobots with chemical sensing and communication capabilities, that searched, targeted and eventually destroyed a tumour. In particular, our scenario's environment includes a number of cancerous cells forming a tumour within a human body. The tumour is located in close proximity to some vascular supply and its approximate location is assumed to be known via existing imaging techniques, therefore an appropriate injection site near the area of interest is presumably selected. A number of nanorobots are subsequently injected to the bloodstream with the purpose of precisely locating the tumour, and then delivering their pharmaceutical payload so as to destroy the tumour cells.

For our simulation, we opted on simulating three different types of simple nanorobots, each group having one main capability. Namely, some "tracking" nanorobots, bearing appropriate chemotactic sensors that can trace the gradient of some chemical marker, some "barrier degrading" nanorobots carrying payload that can help penetrate the vessel wall, and some "drug delivery" nanorobots carrying payload that can destroy the cancerous cells. Our simulated nanorobots are presumed to have limited communication capabilities, that is they are able to send and receive messages and are also limited in energy. Overall, this suggested scenario incorporates the four key features of future nanorobots i.e. power to function, communication, navigation and coordination. A schematic representation of the scenario is shown in Fig. 3.1.

4. Modelling and Design. In order to map the entities described in section 3, the following roles were attributed to three different types of agents: Tracking, Barrier Degrading and Drug Delivery. We used finite state machines extended with memory (X-Machines) [22] to formally design the agents behaviour and plans. The following sections describe the process of formal modelling for our proposed system.

4.1. Formal Modelling with $X$-Machines. An $X$-machine $(\mathcal{X})$ is defined as: $\mathcal{X}=\left(\Sigma, \Gamma, Q, M, \Phi, F, q_{0}\right.$, $\left.m_{0}\right)[20]$, where:

- $\Sigma$ and $\Gamma$ are the input and output alphabets.

- $Q$ is a finite set of states.

- $M$ is a (possibly) infinite set called memory.

- $\Phi$ is a set of partial functions $\varphi$; each such function maps an input, a memory value and an emotional state to an output and a possibly different memory value, $\varphi: \Sigma \times M \times E \rightarrow \Gamma \times M$.

- $F$ is the next state partial function, $F: Q \times \Phi \rightarrow Q$, which given a state and a function from the type $\Phi$ determines the next state. $F$ is often referred to as a state transition diagram.

- $q_{0}$ and $m_{0}$ are the initial state and initial memory.

$\mathrm{X}$-machines are state-based machines extended with a memory structure. This particular feature makes modelling more intuitive and leads towards refinement of the model and eventually implementation. In contrast to memory-less state machines, the transitions between states are not triggered by inputs alone, but by functions that accept an input and the memory values and produce an output and new memory values. Again, this leads nicely towards the final implementation.

There are significant advantages by applying formal modelling mainly concerned with verification and testing. In particular, X-machines have significant advantages over other methods because of their strong legacy of theory and practice in: modelling potential for dynamically structured MAS [47], refinement, animation and simulation [40], testing methods that prove correctness [20] with tools for automatic test generation [11], and model checking for verification of properties [13]. It has been demonstrated that X-Machines and its extensions are particularly useful for modelling biological and biology-inspired MAS [23].

4.2. Simulation Design. Tracking nanorobots (T-bots) are designed as reactive agents, that are initialized in a state of moving randomly. They are able to sense their environment, and more particularly to accept as 


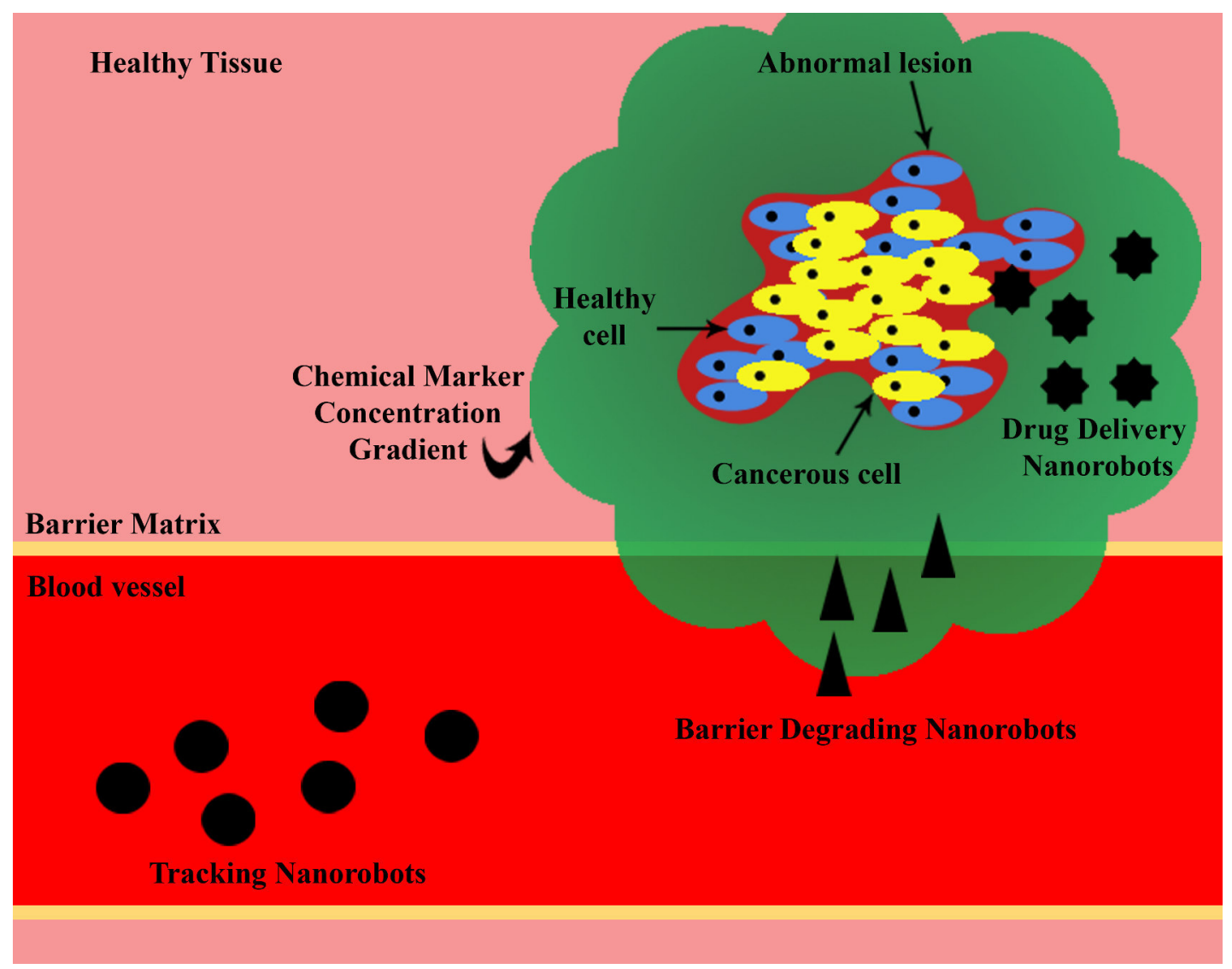

Fig. 3.1: Schematic representation of the targeted drug delivery scenario

input their current location and the protein levels in their surroundings. Once the protein level detected exceeds a predefined value considered as Threshold1, they send a message to a random Barrier Degrading nanorobots (B-bots) in the area, transmitting the corresponding coordinates. They continue moving, until they sense the second protein level value, considered as Threshold2, and then they broadcast a message to all Drug Delivery nanorobots (D-bots) in the area. From initialization and onwards, the T-bots are considered to spend a portion of their initial energy with their every action, namely moving and transmitting messages. Once their initial energy has ran out, the agents die and are removed from the system. The corresponding X-Machine model is depicted in Fig. 4.1. The diagram is an illustration of $F$ (the next state partial function).

The rest of the formal definition falls outside the scope of this paper. However, briefly:

- $\Sigma=\{$ empty_space $:(\mathbb{R}, \mathbb{R})$, protein_level $: \mathbb{R}\}$ is the input alphabet;

- $\Gamma$ is the output alphabet (any actions performed onto the environment and any messages sent to other agents);

- $Q=\{$ moving,protein_detected,dead $\}$ is the set of states.

- $M=((x, y)$, Energy, SensingRadius, ProteinTheshold1, ProteinThreshold 2$)$ is the memory holding the position of the agent, its energy which degrades over time, the radius in which the protein can be detected and the protein thresholds which trigger the message passing.

- $\Phi=\{$ move - randomly, energy - exchausted, detect - protein, continue - searching, inform $-B$ - bots, inform $-D-$ bots $\}$ is the set of functions $\varphi$; for instance the function energy exhausted is defined as: energy - exhausted(empty_space, $((x, y), 0, r$, $b s, d s, t 1, t 2)) \rightarrow($ "outoforder", $((x, y), 0, r, b s, d s, t 1, t 2)))$. Similarly, the rest of the functions are de- 
$M=((x, y)$, Energy, SensingRadius, ProteinThreshold1, ProteinThreshold2)

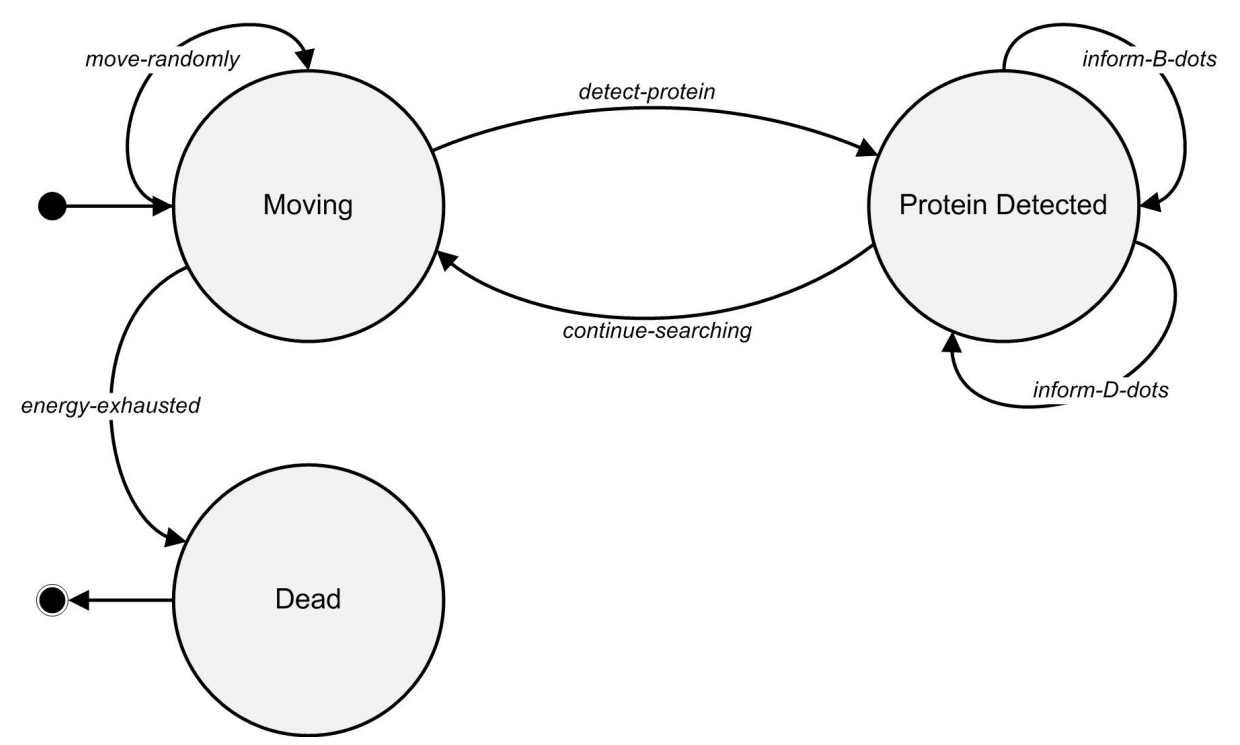

Fig. 4.1: X-Machine model of Tracking nanorobots (T-bots)

fined.

- $q_{0}=$ moving and an example of initial memory might be $m_{0}=((4,8), 15,2, b 1, b 2, b 3$, $d 6, d 7, d 8,0.14,0.17)$ are the initial state and initial memory.

B-bots are initialized as idle, and awaiting for a message from the T-bots. Upon receiving this message, they start moving towards the coordinates that are included in the message they received. Their target location is considered to be near a vessel, since tumours need blood supply to grow and in fact stimulate the growth of new vessels towards them by producing chemical signals, a process known as angiogenesis. Once the $B$-bots reach their target location they move around in a pre-defined radius, searching for a vessel cell. When they detect it, they release their payload and degrade it. Upon doing so, they also broadcast a message to the $D$-bots, transmitting their current location that implies that a pathway has been created for the $D$-bots to pass through and head to the tumour. Similarly with T-bots, the B-bots are assumed to have an initial energy that declines over time with their actions, and once it is exhausted, the agent dies. The corresponding X-Machine model is shown in Fig. 4.2.

Finally, the D-bots are initialized idle and waiting for a message which is initially received by the $B$ bots. Upon receiving the coordinates, the agents move towards the target. By then, the T-bots have also passed through the pathway in the vessel and have accessed the tumour, which means that they have also sent messages with the coordinates where the higher protein level has been detected. Therefore, the D-bots receive a number of target locations, and once again start moving towards them. Their architecture is hybrid, comprising a reactive layer, meaning that if upon moving they come into proximity of a cancerous cell, they release their payload and destroy it. There is also a BDI-type layer, which involves stacking the coordinate data and creating respective goals of reaching the target cell and destroying the cell in location. The corresponding X-machine model is shown in Fig. 4.3.

4.3. Design Constraints and Limitations. Biological systems are inherently complex, and any attempt to model them is accompanied by applying several layers of abstraction. One of the major simplifications of our model is that it does not take into consideration the real environment of the nanorobots in the bloodstream. In reality, the blood itself consists of blood-cells, free macromolecules, etc., elements that are in fact considered to be moving obstacles that the nanorobots should avoid. Additionally, when focusing on the nanoscale, electric 


\section{$M=((x, y)$, Energy, SensingRadius, PayloadCapacity, Goal $)$}

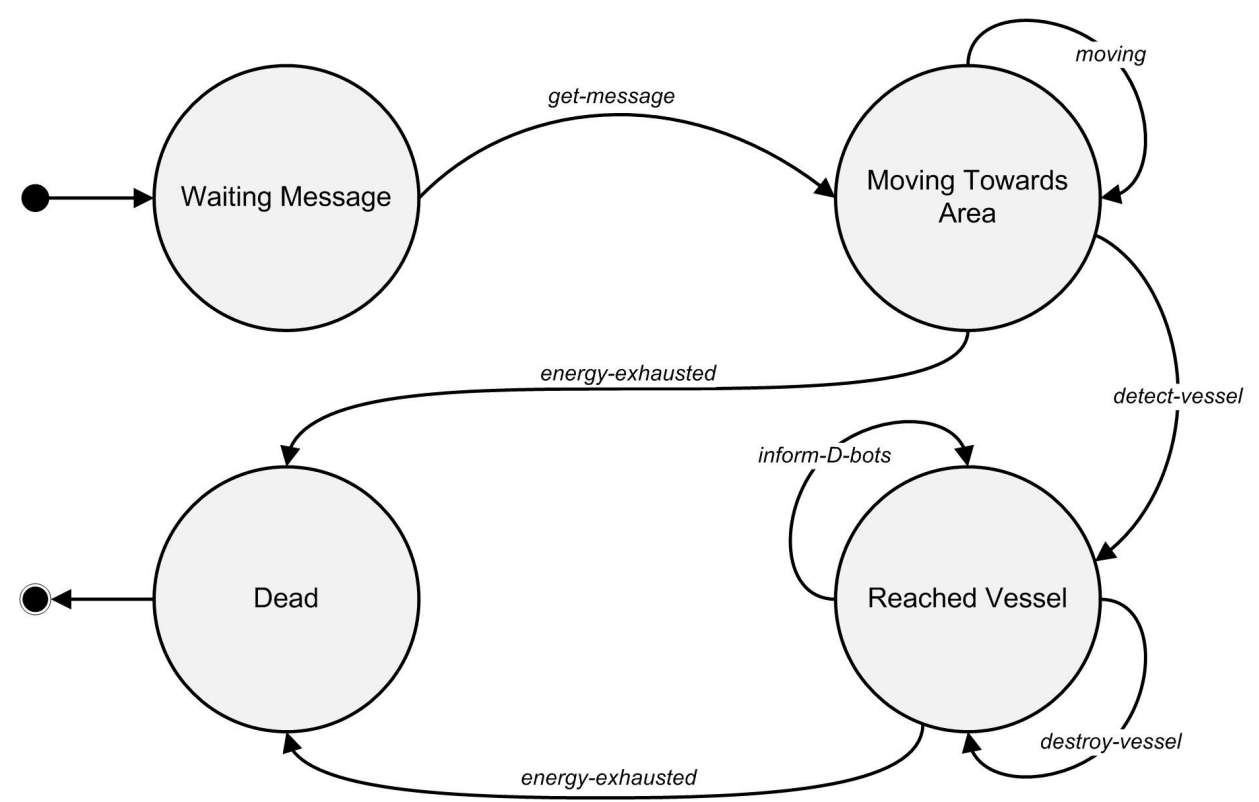

Fig. 4.2: X-Machine model of Barrier Degrading nanorobots (B-bots)

forces are of paramount importance. Finally, when moving in the blood, the nanorobots' movement will be affected greatly by the blood flow.

Furthermore, the physical quantities that are involved in our design, such as energy, time, payload capacity etc. are in our simulation treated as unit-less entities. If such a system was ever to be implemented, all these parameters should be tuned to correspond to some real measurable quantities. This includes any constraints that might be attributed to the limited resources that real nanorobots might have once developed.

Overall, it is important to stress out that our simulation does not attempt to mimic a real nanorobotic drug delivery system, since nanorobots are still theoretical constructs, and there is no way to predict their individual characteristics in a low-detail level. However, our work focuses on demonstrating the applicability of MAS simulations to model a drug delivery system and in doing so, takes into consideration only a limited number of parameters and functionalities.

5. Simulation of our model. The rapid growth of Agent Based Modelling is accompanied by an increase in the number of software platforms that are utilized for building agent based simulations [2]. Some of the most popular ones are Swarm [33], Repast [36], MASON [28], FLAME [9] and NetLogo [54], the latter being our selected choice amongst the rest of candidates because it is simple and powerful enough to meet our aims. Our choice would have been different if the simulation was meant to increase in scale and functionality.

NetLogo is both a programming language and a programmable modelling environment for simulating multiagent systems, with a built-in graphical user interface for immediate visualization of the simulation. Languagewise, NetLogo supports agents with a rather extensive vocabulary of built-in primitives. Our criteria for selecting NetLogo as the development framework include the following:

- Given that the vast majority of our system's functional requirements involve spatial movement, the most fundamental consideration was to utilize a platform that would natively support spatial agent characteristics.

- Based on the user requirements, manipulating the model's parameters and collecting the simulation results should be accomplished in a user-friendly manner. 


\section{$M=((x, y)$, Energy, SensingRadius, PayloadCapacity, Goal)}

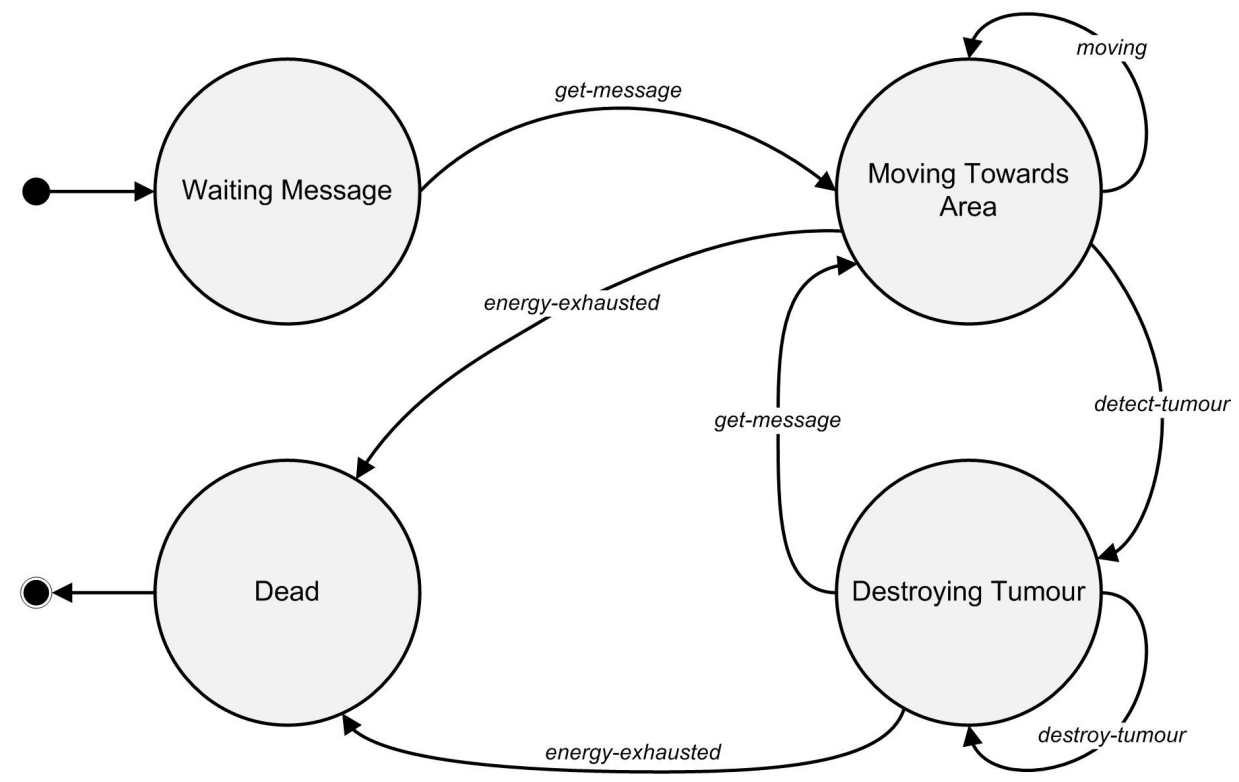

Fig. 4.3: X-Machine model of Drug Delivery nanorobots (D-bots)

- Availability of runtime visualization was desirable, since this would provide the additional benefit of observing behaviours that are not necessarily reflected on the numerical data collection.

- Agent communication should also be supported, as well as the implementation of both reactive and proactive agent architectures.

NetLogo is a platform primarily intended for constructing social and natural phenomena models, it thus meets the first three criteria but does not support neither agent communication nor proactive architectures [45]. Two NetLogo libraries [44] were used, one that includes procedures and reporters tailored to implement message passing, and thus agent communication may be achieved and one for implementing some form of proactive behaviour. The former library includes primitives for creating, sending and receiving a message that follows closely the FIPA-ACL [50] message format.

6. Results and Experimental Analysis. The simulation graphical interface is presented in Fig. 6.1. A simple experiment was conducted. In a scenario closely mapped to Fig. 3.1, the environment was set-up with the upper area representing the body tissue, the middle (brown) area representing the vessel wall and the bottom (red) area representing the blood within the vessel. Within the body tissue area, a number of tumourcells (purple) depicting the tumour were created in a random position, and the remaining area was filled with body-cells (pink). Subsequently, the area surrounding the tumour was given a protein concentration property, with a value gradually declining with distance from the tumour walls. A user-defined number of each type of agents, namely Tracking T-bots, Barrier Degrading B-bots and Drug Delivery D-bots, were randomly initiated in the bottom left side of the simulation area, which was selected to represent the injection site. Three possible experiment outcomes were identified:

1. Tumour eliminated: no more tumour cells left in the simulation world;

2. Failure to eliminate tumour: no more D-bots left but at least one tumour cell exists;

3. Failure to complete experiment: one or more $D$-bot fail to transition from idle state because all $B$-bots fail to degrade even one vessel cell.

By using the integrated NetLogo BehaviorSpace tool, a number of experiments were executed for different sets of parameter values, and with 500 simulation runs for each experiment. Although normally a thorough 


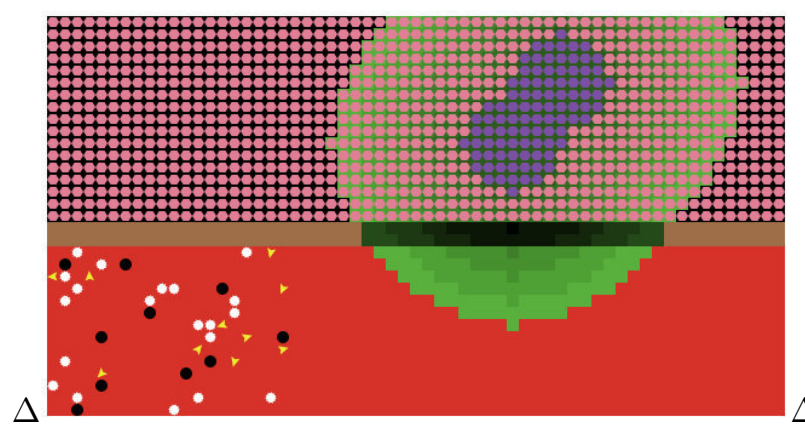

() Initial Setup

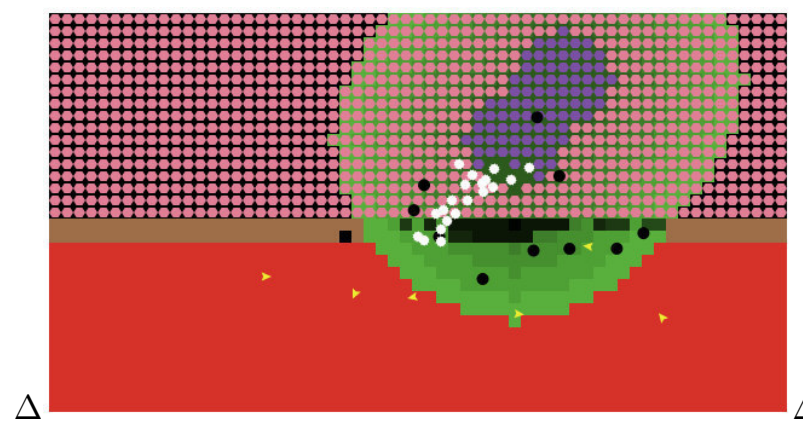

() D-bots destroying tumour cells

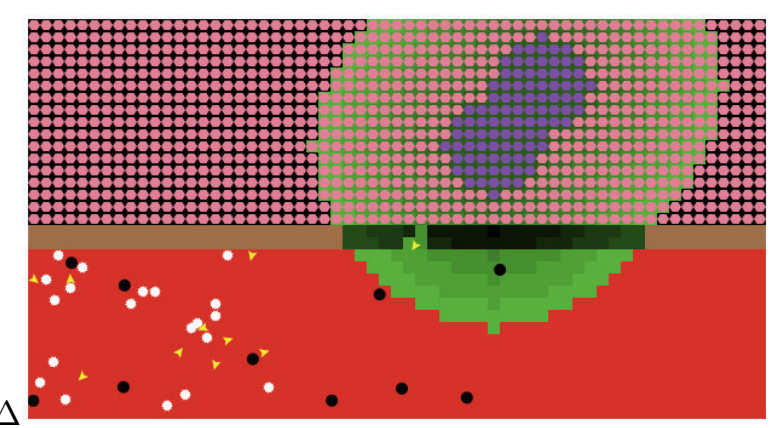

() A B-bot degrades vessel cell

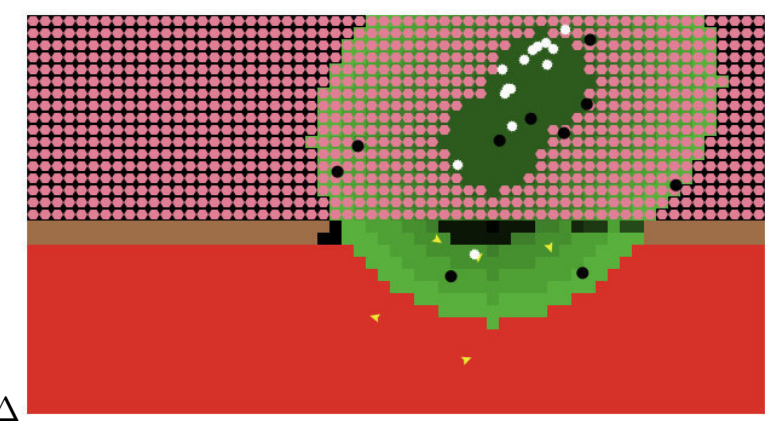

() Tumour elimination

Fig. 6.1: Simulation Interface: The graphical interface developed to run the simulation

statistical analysis would be required, at these early stages we decided to run simple experiments in order to visualize the agent models created as well as to verify certain aspects of the experiments which were intuitively predicted. The stochastic elements in these experiments are simply based on the random setup of the agents as well as on their random move. Apart from this, individual agents were designed to operate in a deterministic way which however in collaboration results in an emergent behaviour.

The experiments were grouped into four categories, one for each set of parameters, i.e. number of $T$-bots, number, payload capacity and sensing radius of $B$-bots, number and payload capacity of $D$-bots and energy. The purpose was to estimate how different values for each of these entities would result in one of the three possible outcomes. For each experiment in a group, the relative frequencies of occurrence of the three possible end-results were calculated.

The results were qualitatively assessed and interpreted under two perspectives. On one hand, some conclusions were drawn that indicate the effect of the parameter values on the designed simulation outcome. On the other hand, the results were considered as likely offering some insight on how to improve the design of the system, hence, produce a more efficient or error-free model. Lacking any real-life experimental data to compare our results with, the model itself was validated against the main objective of tumour destruction.

The first conclusion that was drawn is that the system was able to accomplish the set goal of tumour elimination. As the results indicated, even with a low numbers of $T$-bots and B-bots, and even with a low $D$-bot payload capacity and sensing radius, it was still possible to eliminate the tumour. Nevertheless, the highest percentages of tumour elimination were achieved for high numbers of active agents. Various experiments have verified the intuition one might have about the importance of the three parameters in the simulations. For instance, failure to eliminate the tumours is related to the number of agents of each type. This is apparent when we incrementally increase the number of agents of each type, as shown in Fig. 6.2. However, the results indicate that the effect of increasing numbers of $T$-bots and $B$-dots becomes important for low numbers of 
agents. The corresponding curves reach a plateau, and numbers higher than do not seem to lead to higher success percentages. Similarly, regarding the D-dots, a minimum threshold appears to exist, in the sense that with less than 8 D-bots the percentages of successful outcomes were close to zero. A steep increase appears after above between 8 and 10 D-bots, and once again, a plateau seems to emerge for slightly higher numbers. This observation suggests that there is a narrow band of appropriate values for the number of $D$-bots. Naturally, this observation is strongly correlated to the specific model, however, this phenomenon is a fitting example of how MAS simulations may offer advantageous insight into better understanding and possibly improving complex systems.

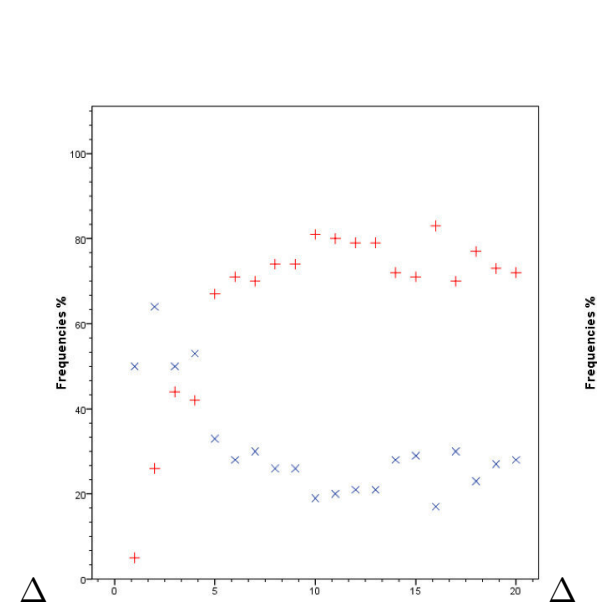

() T-bots
+ Tumour Eliminated

$\times$ Failed To Eliminate Tumour

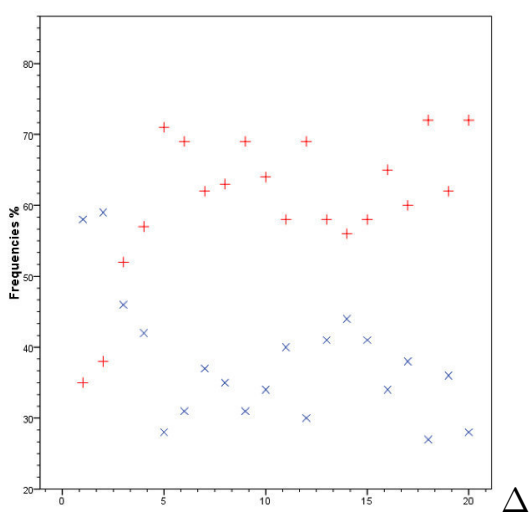

() B-bots

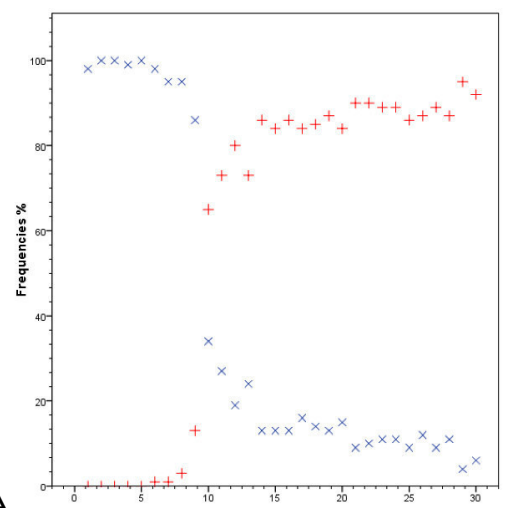

() D-bots

Fig. 6.2: Frequencies of outcomes plotted against numbers of agents

Further observations include:

- The effects of the $B$-bot sensing radius and payload capacity and the $D$-bot payload capacity were investigated. Regarding the $B$-bots, it appeared that even with a maximum value for their number, the percentage of tumour elimination is very low. This was attributed to the fact that - in the specific design - for the B-bots to penetrate the vessel wall they needed to degrade two consecutive vessel cells. With the payload capacity and the sensing radius set at the minimum value, tumour elimination was an extremely rare occurrence.

- It was also estimated that the $B$-bot payload parameter affected the outcome substantially more than the sensing radius. Indeed, when the payload was minimum, even with a high sensing radius, the "Tumour eliminated" outcome was quite rare, with recorded frequencies ranging from $1.83 \%$ to $3.33 \%$. On the contrary, a minimum percentage of $15 \%$ was recorded when the payload was set to a medium value. This effect, however, is not observed for the maximum value for the sensing radius, implying that its significance lessens from a certain point onwards. Even with both parameters set on their highest values, tumour elimination was recorded in roughly $65 \%$ of the cases. This result, compared to the percentages of the control experiments (medium values for all parameters), supports the argument that the number of $D$-bots affects the outcome more strongly than the $B$-bot payload and sensing radius.

- The D-bot payload appeared to contribute greatly to the outcome, where an increase reaching almost $88 \%$ for successful tumour elimination was observed when the corresponding parameter was set from low to medium. However, this increase became minimal between medium and high values for the same parameter, implying that its contribution had reached a plateau. This observation could offer insight on determining the optimum payload capacity, should such a system be realized in real-life.

- A set of experiments were designed specifically to estimate the effect of the energy parameter, that would be of special interest should such a system ever exist in reality. The results are shown in Fig. 6.3. The 
percentage frequencies of occurrence of the "Tumour Eliminated" and "Failed to Eliminate Tumour" outcomes were plotted against the corresponding values of the energy parameter, while maintaining average numbers. A correlation may be observed between the two sets of variables, indicating that as the energy values were incrementally increased, the probability for tumour elimination increased as well, whereas the probability for failure to eliminate the tumour decreased. Additionally, for energies lower than 100, the agents could not achieve tumour elimination, whereas for energies higher than 600 , the percentage of success seems to remain unaffected. Once again, this indicates that MAS simulations could possibly be used to identify lower and upper limits in the values of parameters of interest, should such a system ever be realized. The line for the "Failure to Complete Experiment" outcome is not plotted, as it has no practical value. However, the percentage of its occurrence may be directly deduced from the graph.

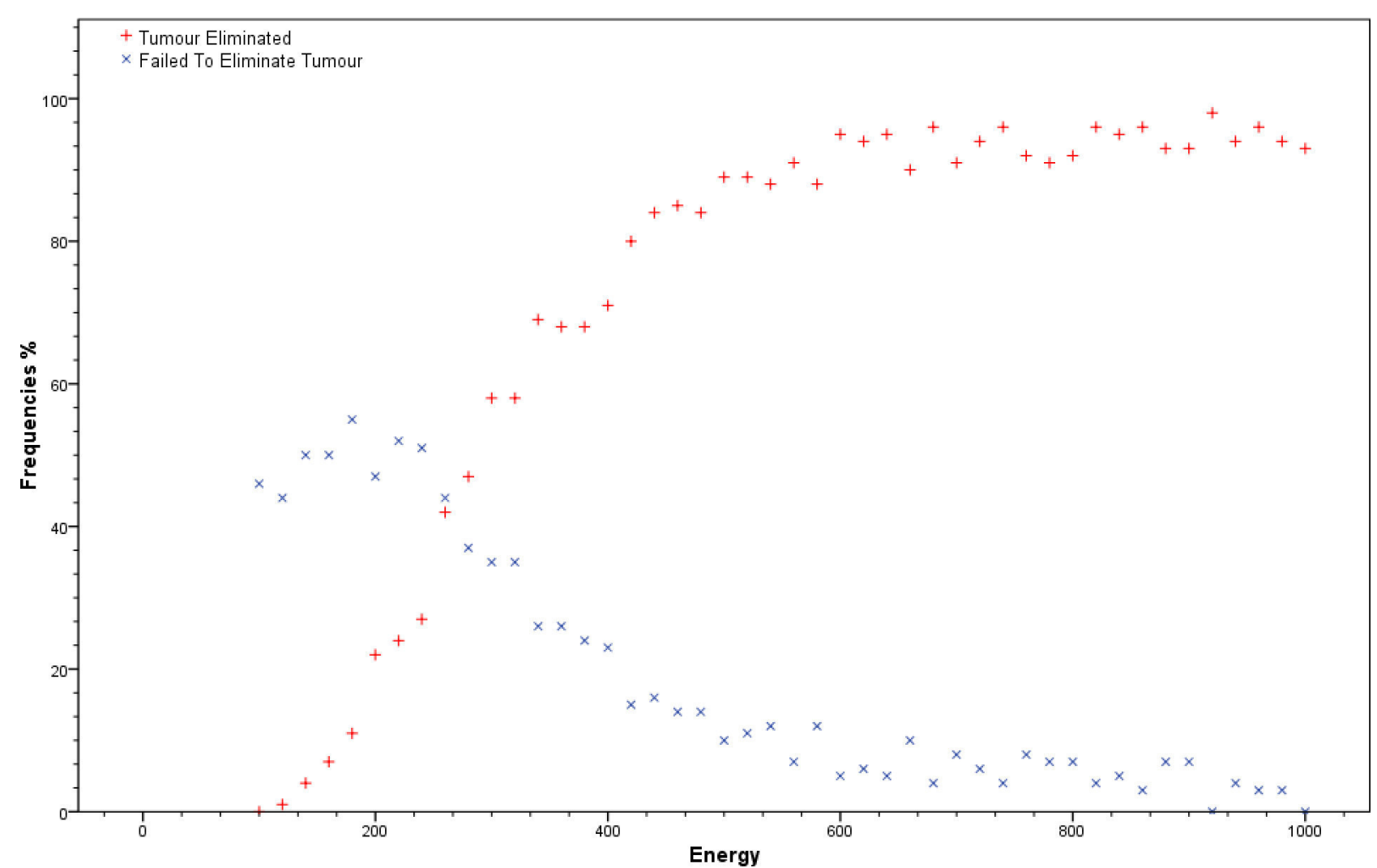

Fig. 6.3: Frequencies of Tumour Eliminated and Failed To Eliminate Tumour outcomes plotted against the energy values.

Regarding the system's overall functionality, the experiments served as an indirect validation against the set requirements. A successful outcome, i.e. a "Tumour eliminated" result was reproducibly achievable. The observed "Failed to complete experiment" high percentage in experiments with all parameters set to their lowest values was anticipated. It was a design decision to favour energy preservation over experiment completion concerns, by initializing both B-bots and D-bots awaiting for a message before exiting the initial idle state. This lead to the possibility of these agents staying inactive, in the case that not enough T-bots managed to sense the protein gradient. Nevertheless, the observed percentages in subsequent experiments with high values for the agent parameters, suggest that a better design should perhaps be considered, to combine both energy preservation with optimum experiment completion occurrences. 
7. Nanorobotics: Simulation versus Reality. Currently, the nanorobots as envisaged in our system, are purely theoretical artifacts. Nanorobots are a distant and ambiguous concept. However, researchers involved in the domain insist that studying and attempting to mimic nature's molecular machines, which have been working and optimizing their functionalities over millions of years, might be the best approach towards fabricating real nanorobots. A roadmap proposed in [30] describes the following steps, yet far from reality:

- Developing bio-nanocomponents, such as bio-nanosensors and bio-nanomotors;

- Assembling the bio-nanocomponents into bio-nanorobots;

- Developing the concepts for nanorobot collaboration (bionanoswarms), establishing the methods for distributive intelligence, programming and control;

- Developing and implementing automatic fabrication methodologies.

Technology nowadays allows for fabrication of micro- rather than nano-components. Spiral-shaped devices with lengths of approximately $50 \mu \mathrm{m}$, that are able to swim in a controlled fashion under weak magnetic field application have already been constructed [55]. Fabrication of a prototype autonomous robot of size $3.9 \times 3.9 \times$ $3.3 \mathrm{~mm}^{3}$, with communication, locomotion and energy storage capabilities is possible [12]. The first controlled vertical flight of a $19 \mathrm{~mm}$ sized microrobot has been announced [39]. These technological advances are merely mentioned to report the current status of microrobotic fabrication, indicating that manufacturing capabilities become more limited as size decreases.

However, what is of significant interest from a MAS viewpoint is the prospects of nanoro-botic communication and collaboration. To achieve nanorobotic collaboration, strategies for communication among nanorobots must be designed. Communication by means of acoustic waves has been recently investigated by Hogg and Freitas, and findings were reported in [18]. Spherically shaped robots of three different radii; $0.5 \mu \mathrm{m}$ for the size of an isolated nanorobot, $5 \mu \mathrm{m}$ for nanorobot aggregates that would address tasks requiring greater than individual capabilities, and $50 \mu \mathrm{m}$ for the size of tissue-embedded signal repeater stations were mathematically investigated. It was concluded that communication could occur at $10^{4} \mathrm{bits} / \mathrm{s}$, over distances of $100 \mu \mathrm{m}$, depending on available power and on safety constraints (i.e. nanorobot heating).

Another approach towards communication is once again inspired by nature, namely chemical signalling. This method for communication has been observed both in the macro-world (ants that release and sense pheromone trails [21]) and in the microworld (bacteria that produce and respond to signalling molecules called autoinducers, in a process called quorum sensing [49]).

The issue of coordination will require far more elaborate mechanisms. Swarm intelligence may currently be investigated only via computer simulations. The decentralized movement coordination by implementing an enhanced version of common flocking algorithms is investigated [1]. A framework based on an adopted variation of the Artificial Bee Colony algorithm is also proposed [35]. It is evident that the actual fabrication of nanorobot-swarms will require the realisation of individual nanorobot assembly first, but researchers are committed to explore such models in anticipation of the technological breakthrough.

8. Conclusions and Future Work. We demonstrated how Multi-Agent Systems can assist in simulating drug delivery in unhealthy tissues by a set of collaborating nanorobots. Despite the fact that such a system is far yet from becoming reality, MAS appeared to be an applicable solution in modelling and identifying a number of key-issues. A targeted drug delivery system was designed, including formal models of agents that work together in order to treat a tumour located inside some bodily tissue. A simulation based on the formal models was developed in order to demonstrate how various parameters affect the overall treatment.

Further work would include improvement on the design of MAS. This could include enhancing the agent communication, from simple message passing to implementing some existing agent interaction protocols, i.e. introducing some form of negotiation. Another improvement includes refining the parameters to be investigated in a more pragmatic manner. Blood elements might be added as obstacles in the nanorobot movement, and blood flow may also be considered. Furthermore, inclusion of physical properties, such as blood viscosity, tissue density and electric current can result in a more detailed system. 


\section{REFERENCES}

[1] G. Al-HudHud, On Swarming Medical Nanorobots, International Journal of Bio-Science and Bio-Technology, 4 (2012), pp. 7590.

[2] R. J. Allan, Survey of agent based modelling and simulation tools, Tech. Report DL-TR-2010-007, Science and Technology Facilities Council, oct 2010.

[3] R. A. Brooks, Intelligence without reason, in Proceedings of the 12th International Joint Conference on Artificial Intelligence (IJCAI-91), J. Myopoulos and R. Reiter, eds., Sydney, Australia, 1991, Morgan Kaufmann publishers Inc.: San Mateo, CA, USA, pp. 569-595.

[4] — Intelligence without representation., Artificial Intelligence, 47 (1991), pp. 139-159.

[5] A. Cavalcanti, L. Rosen, L. Kretly, M. Rosenfeld, and S. Einav, Nanorobotic challenges in biomedical applications, design and control, in Electronics, Circuits and Systems, 2004. ICECS 2004. Proceedings of the 2004 11th IEEE International Conference on, dec. 2004, pp. $447-450$.

[6] A. Cavalcanti, L. Rosen, B. Shirinzadeh, M. Rosenfeld, S. Paulo, and T. Aviv, Nanorobot for treatment of patients with artrery occlusion, Platelets, 20 (2007), pp. 1-10.

[7] A. Cavalcanti, B. Shirinzadeh, T. Fukuda, and S. Ikeda, Nanorobot for brain aneurysm, The International Journal of Robotics Research, 28 (2009), pp. 558-570.

[8] A. Cavalcanti, B. Shirinzadeh, D. Murphy, and J. A. Smith, Nanorobots for laparoscopic cancer surgery, in Computer and Information Science, ACIS International Conference on, IEEE Computer Society, 2007, pp. 738-743.

[9] S. Chin, FLAME (FLexible Large-scale Agent Modelling Environment), 2014.

[10] M. Coccia, U. FinARDI, AND D. MARGON, Current trends in nanotechnology research across worldwide geo-economic players, The Journal of Technology Transfer, 37 (2012), pp. 777-787.

[11] D. Dranidis, K. Bratanis, and F. Ipate, JSXM: A tool for automated test generation, in Software Engineering and Formal Methods, Springer, 2012, pp. 352-366.

[12] E. Edqvist, N. Snis, R. C. Mohr, O. Scholz, P. Corradi, J. Gao, A. Diguez, N. Wyrsch, and S. Johansson, Evaluation of building technology for mass producible millimetre-sized robots using flexible printed circuit boards, Journal of Micromechanics and Microengineering, 19 (2009), p. 075011.

[13] G. Eleftherakis and P. Kefalas, Model checking safety critical systems specified as X-machines, Matematica-Informatica, Analele Universitatii Bucharest, 49 (2000), pp. 59-70.

[14] Fluent INC., FLUENT. http://www.fluent.com.

[15] R. A. Freitas, Pharmacytes: an ideal vehicle for targeted drug delivery, Journal of Nanoscience and Nanotechnology, 6 (2006), pp. $2769-75$.

[16] T. Hamaguchi, T. Doi, T. Eguchi-Nakajima, K. Kato, Y. Yamada, Y. Shimada, N. Fuse, A. Ohtsu, S.-I. Matsumoto, M. TAkanashi, And Y. Matsumura, Phase I Study of NK012, a Novel SN-38Incorporating Micellar Nanoparticle, in Adult Patients with Solid Tumors, Clinical Cancer Research, 16 (2010), pp. 5058-5066.

[17] E. J. Hanly, M. R. Marohn, S. L. Bachman, M. A. Talamini, S. O. Hacker, R. S. Howard, and N. S. Schenkman, Multiservice laparoscopic surgical training using the davinci surgical system, American Journal of Surgery, 187 (2004), pp. 309-315.

[18] T. Hogg And R. A. F. JR.), Acoustic communication for medical nanorobots, Nano Communication Networks, 3 (2012), pp. $83-102$.

[19] O. E. Holland and C. R. Melhuish, Getting the most from the least: Lessons for the nanoscale from minimal mobile agents, in Artificial Life V: Proceedings of the Fifth International Workshop on the Synthesis and Simulation of Living Systems, C. G. Langton and K. Shimohara, eds., MIT Press, 1997, pp. 59-66.

[20] F. Ipate And M. Holcombe, An integration testing method that is proved to find all faults, International Journal of Computer Mathematics, 63 (1997), pp. 159-178.

[21] D. E. Jackson and F. L. W. Ratnieks, Communication in ants., Current biology : CB, 16 (2006), pp. R570-4.

[22] P. Kefalas, M. Holcombe, G. Eleftherakis, and M. Gheorghe, Intelligent Agent Software Engineering, IGI Global, July 2002.

[23] P. Kefalas, I. Stamatopoulou, I. Sakellariou, and G. Eleftherakis, Transforming Communicating X-machines into P Systems, Natural Computing, 8 (2009), pp. 817-832.

[24] J. King, M. Lees, and B. Logan, Agent-based and continuum modelling of populations of cells, December 2006.

[25] A. Kumari, S. K. Yadav, and S. C. Yadav, Biodegradable polymeric nanoparticles based drug delivery systems, Colloids and Surfaces B: Biointerfaces, 75 (2010), pp. $1-18$.

[26] D. L. Leslie-Pelecky and R. D. Rieke, Magnetic properties of nanostructured materials, Chemistry of Materials, 8 (1996), pp. $1770-1783$.

[27] L. Li, F. Tang, H. Liu, T. Liu, N. HaO, D. Chen, X. Teng, and J. He, In vivo delivery of silica nanorattle encapsulated docetaxel for liver cancer therapy with low toxicity and high efficacy, ACS Nano, 4 (2010), pp. 6874-6882.

[28] S. Luke, C. Cioffi-Revilla, L. Panait, K. Sullivan, and G. Balan, MasON: A multiagent simulation environment, SIMULATION, 81 (2005), pp. 517-527.

[29] J. Martín, J. Nogués, K. Liu, J. Vicent, and I. K. Schuller, Ordered magnetic nanostructures: fabrication and properties, Journal of Magnetism and Magnetic Materials, 256 (2003), pp. $449-501$.

[30] C. Mavroidis, A. Ummat, and A. Dubey, Bio-Nanorobotics, in Biomimetics, CRC Press, Nov. 2005, pp. $201-227$.

[31] A. D. McCulloch and G. Huber, Integrative Biological Modelling In Silico, John Wiley \& Sons, Ltd, 2008, pp. 4-25.

[32] C. Melhuish And J. Welsby, Gradient ascent with a group of minimalist real robots: implementing secondary swarming, in Systems, Man and Cybernetics, 2002 IEEE International Conference on, vol. 2, Oct 2002, pp. 509-514. 
[33] N. Minar, R. Burkhart, C. Langton, and M. Askenazi, The Swarm simulation system: A toolkit for building multi-agent simulations, Working Papers 96-06-042, Santa Fe Institute, June 1996.

[34] Nano.gov, Benefits and applications - nano. http://www.nano.gov/you/nanotechnology-benefits, 2014. On-line; Accessed October 20, 2014.

[35] T. Nantapat, B. Kaewkamnerdpong, T. Achalakul, and B. Sirinaovakul, Best-so-far ABC based nanorobot swarm, in Proceedings of the 2011 Third International Conference on Intelligent Human-Machine Systems and Cybernetics Volume 01, IHMSC '11, Washington, DC, USA, 2011, IEEE Computer Society, pp. 226-229.

[36] M. North, T. Howe, N. Collier, AND J. Vos, A declarative model assembly infrastructure for verification and validation, in Advancing Social Simulation: The First World Congress, S. Takahashi, D. Sallach, and J. Rouchier, eds., Springer Japan, 2007, pp. 129-140.

[37] M. Ntika, P. Kefalas, and I. Stamatopoulou, Multi-agent system simulation of nano-robotic drug delivery in tumours of body tissues, in System Theory, Control and Computing (ICSTCC), 2013 17th International Conference, Oct 2013, pp. $777-782$.

[38] G. M. Patel, G. C. Patel, R. B. Patel, J. K. Patel, and M. Patel, Nanorobot: a versatile tool in nanomedicine., Journal of Drug Targeting, 14 (2006), pp. 63-67.

[39] N. O. Pérez-Arancibia, K. Y. Ma, K. C. Galloway, J. D. Greenberg, and R. J. Wood, First controlled vertical flight of a biologically inspired microrobot, Bioinspiration \& Biomimetics, 6 (2011), p. 036009.

[40] I. Petreska, P. Kefalas, and M. Gheorghe, A framework towards the verification of emergent properties in spatial multiagent systems, Proceedings of the Workshop on Applications of Software Agents, (2011), pp. 37-44.

[41] C. A. Piña García, E. J. Rechy-Ramírez, and V. A. García-Vega, Using an alternative model in a complex environment for nanorobotics navigation, in 16th International Conference on Computing (CIC), nov. 2007.

[42] - Comparing three simulated strategies for cancer monitoring with nanorobots, in MICAI 2008: Advances in Artificial Intelligence, A. Gelbukh and E. F. Morales, eds., vol. 5317 of Lecture Notes in Computer Science, Springer Berlin Heidelberg, 2008, pp. 1020-1030.

[43] H. Redhead, S. Davis, And L. Illum, Drug delivery in poly(lactide-co-glycolide) nanoparticles surface modified with poloxamer 407 and poloxamine 908: in vitro characterisation and in vivo evaluation, Journal of Controlled Release, 70 (2001), pp. $353-363$.

[44] I. Sakellariou, P. Kefalas, and I. Stamatopoulou, Enhancing NetLogo to simulate BDI communicating agents, in Proceedings of the 5th Hellenic conference on Artificial Intelligence: Theories, Models and Applications, SETN '08, Berlin, Heidelberg, 2008, Springer-Verlag, pp. 263-275.

[45] I. Sakellariou, P. Kefalas, and I. Stamatopoulou, Teaching intelligent agents using NetLogo, in Proceedings of the Informatics Education Europe III Conference (IEEIII 2008), A. Cortesi and F. Luccio, eds., 2008, pp. 209-221.

[46] G. Sharma, C. Mavroidis, and A. Ferreira, Virtual reality and haptics in nano- and bionanotechnology, in Handbook of Theoretical and Computational Nanotechnology, M. Rieth and W. Schommers, eds., vol. X, American Scientific Publishers, 2005, ch. 40, pp. 1-33.

[47] I. Stamatopoulou, P. Kefalas, and M. Gheorghe, OPERAS: a formal framework for multi-agent systems and its application to swarm-based systems, in Proceedings of the 8th International Workshop on Engineering Societies in the Agents World (ESAW'07), A. Artikis, G. O'Hare, K. Stathis, and G. Vouros, eds., 2007, pp. 208-223.

[48] B. STEELE, 'Ordered' catalyst boosts fuel cell output at lower cost. http://www . nanowerk. com/news2/newsid=27135.php, 2012. [Online; accessed 20-April-2014].

[49] M. E. Taga and B. L. Bassler, Chemical communication among bacteria, Proceedings of the National Academy of Sciences of the United States of America, 100 (2003), pp. 14549-14554.

[50] The Foundation for Intelligent Physical Agents, FIPA, The history of FIPA. http://www.fipa.org/subgroups/ ROFS-SG-docs/History-of-FIPA.htm. On-line; Accessed November 11, 2012.

[51] A. Trafton, Super-sensitive and small: New MIT detector uses nanotubes to sense deadly gases. http://newsoffice.mit. edu/2008/nanotube-0605, 2008. [Online; accessed 20-April-2014].

[52] A. Ummat, G. Sharma, A. Dubey, and C. Mavroidis, Bio-nanorobotics: State of the art and future challenges, in Tissue Engineering and Artificial Organs, The Biomedical Engineering Handbook, J. D. Bronzino, ed., London, UK: CRC Press, 2006, ch. 19, pp. 1-42.

[53] R. VALIEV, Nanostructuring of metals by severe plastic deformation for advanced properties, Nature materials, 3 (2004), pp. 511-516.

[54] U. WILENSKY, NetLogo. http://ccl.northwestern.edu/netlogo/, 1999. Center for Connected Learning and Computer-Based Modeling, Northwestern University. Evanston, IL.

[55] L. Zhang, J. J. Aвbott, L. Dong, B. E. Kratochvil, D. Bell, and B. J. Nelson, Artificial bacterial flagella: Fabrication and magnetic control, Applied Physics Letters, 94 (2009), pp. 064107 -064107-3.

Edited by: Costin Bădică

Received: July 21, 2014

Accepted: Sept 21, 2014 